\title{
Multiple calcified right ventricular masses presenting with pulmonary embolism and severe pulmonary hypertension
}

\author{
Ji-Yong Jang, MD, ${ }^{a}$ Byung-Chul Chang, MD, PhD, ${ }^{\mathrm{b}}$ and Chi Young Shim, MD, PhD, ${ }^{\mathrm{a}}$ Seoul, Republic \\ of Korea
}

Intracardiac calcified masses may represent neoplastic or nonneoplastic processes. Most cardiac neoplastic disorders are benign, such as myxomas. ${ }^{1}$ Nonneoplastic disorders include mural thrombi and infectious processes, such as endocarditis. We report a case of multiple calcified right ventricular masses in a young man, probably resulting from an episode of blunt chest trauma, and subsequent chronic thrombus that appeared as pulmonary embolism and severe pulmonary hypertension.

\section{CLINICAL SUMMARY}

A 34-year-old man was admitted for shortness of breath (New York Heart Association class II), edema of both legs, and abdominal distention of 3 years' duration. Two years earlier he had been diagnosed with congestive hepatopathy. He denied alcohol or drug use and had no evidence of viral hepatitis. There was no history of thromboembolic, hematologic, or malignant disease. However, at the age of 30 years, he had a head-on collision with someone and was kicked on the anterior part of the chest while water skiing.

Echocardiography revealed enlarged right-sided chambers and multiple calcified masses involving the right ventricle and the subvalvular apparatus of the tricuspid valve (Figure 1, A). However, right ventricular systolic function was preserved, as assessed by tissue Doppler imaging of the lateral tricuspid annular motion. Color Doppler imaging showed severe tricuspid regurgitation (Figure 1,B) combined with severe pulmonary hypertension (Figure 1,C). A computed tomographic scan of the chest demonstrated a conglomerated calcified mass at the right ventricle and calcified emboli at both pulmonary arteries (Figure 1,D). Cardiac magnetic resonance imaging suggested a calcific amorphous mass rather than cardiac myxoma or fibroma (Figure 1,E).

Despite 1 year of medical treatment, the symptoms persisted. Therefore, he underwent an open cardiac operation.

From the Cardiology Division ${ }^{\mathrm{a}}$ and the Division of Cardiovascular Surgery, ${ }^{\mathrm{b}}$ Severance Cardiovascular Hospital, Yonsei University College of Medicine, Seoul, Republic of Korea.

Disclosures: None.

Received for publication April 13, 2010; revisions received May 3, 2010; accepted for publication May 14, 2010; available ahead of print June 16, 2010.

Address for reprints: Chi Young Shim, MD, PhD, Cardiology Division, Severance Cardiovascular Hospital, Yonsei University College of Medicine, Seongsanno 250, Seodaemun-Gu, Seoul, Republic of Korea 120-752 (E-mail: cysprs@yuhs.ac). J Thorac Cardiovasc Surg 2010;140:e64-5

$0022-5223 / \$ 36.00$

Copyright (c) 2010 by The American Association for Thoracic Surgery doi:10.1016/j.jtcvs.2010.05.029
Multiple calcified masses were removed from the right ventricle and pulmonary arteries as much as possible. Because some calcified masses were firmly attached to the vessel wall, especially the pulmonary artery, some masses could not be removed. Tricuspid valve repair was performed with an annuloplasty ring. The gross specimen showed multiple pieces of calcified masses with an irregular structure (Figure 1,F), and histopathologic examination revealed dense fibrosis and calcification (Figure 1, G). After surgery, the patient's symptoms were well controlled in New York Heart Association class I, and liver function was normalized. To date, follow-up has been performed in our outpatient clinic for 4 months and the follow-up echocardiogram revealed a decrease in right ventricular size and preservation of right ventricular systolic function.

\section{DISCUSSION}

Regardless of its size or location, intracardiac mural thrombus may be challenging to diagnose. It is especially difficult to distinguish chronic calcified intracardiac thrombus from other causes of intracardiac calcification, particularly tumor. Because the symptoms are not distinct from those of other conditions, the patient's history may reveal the masking clue.

Blunt chest trauma can cause right ventricular contusion because of the location of the right ventricle and its proximity to the anterior chest wall and sternum. It is likely that chest trauma caused myocardial contusion with impaired right ventricular systolic function and perhaps endocardial damage. As a result of hemodynamic stasis of blood flow in the right ventricle, thrombus was subsequently formed. In such cases, echocardiography may demonstrate wall motion abnormality, right ventricular dilatation, myocardial thinning, and intracavitary thrombus. ${ }^{2}$ During the 3 -year time interval between chest trauma and the diagnosis of multiple right ventricular thrombi, dense calcifications could be formed. Such nonneoplastic cardiac calcified masses are described as calcified amorphous tumors in the pathology literature. ${ }^{3}$

This patient had multiple intracavitary calcified masses involving the right ventricle and the subvalvular apparatus of the tricuspid valve. Moreover, calcified masses were also found in the main pulmonary artery. It is more likely that multiple calcified masses embolize and obstruct pulmonary arteries than that a calcified mass forms de novo in the pulmonary arteries. The obstruction of pulmonary arteries 

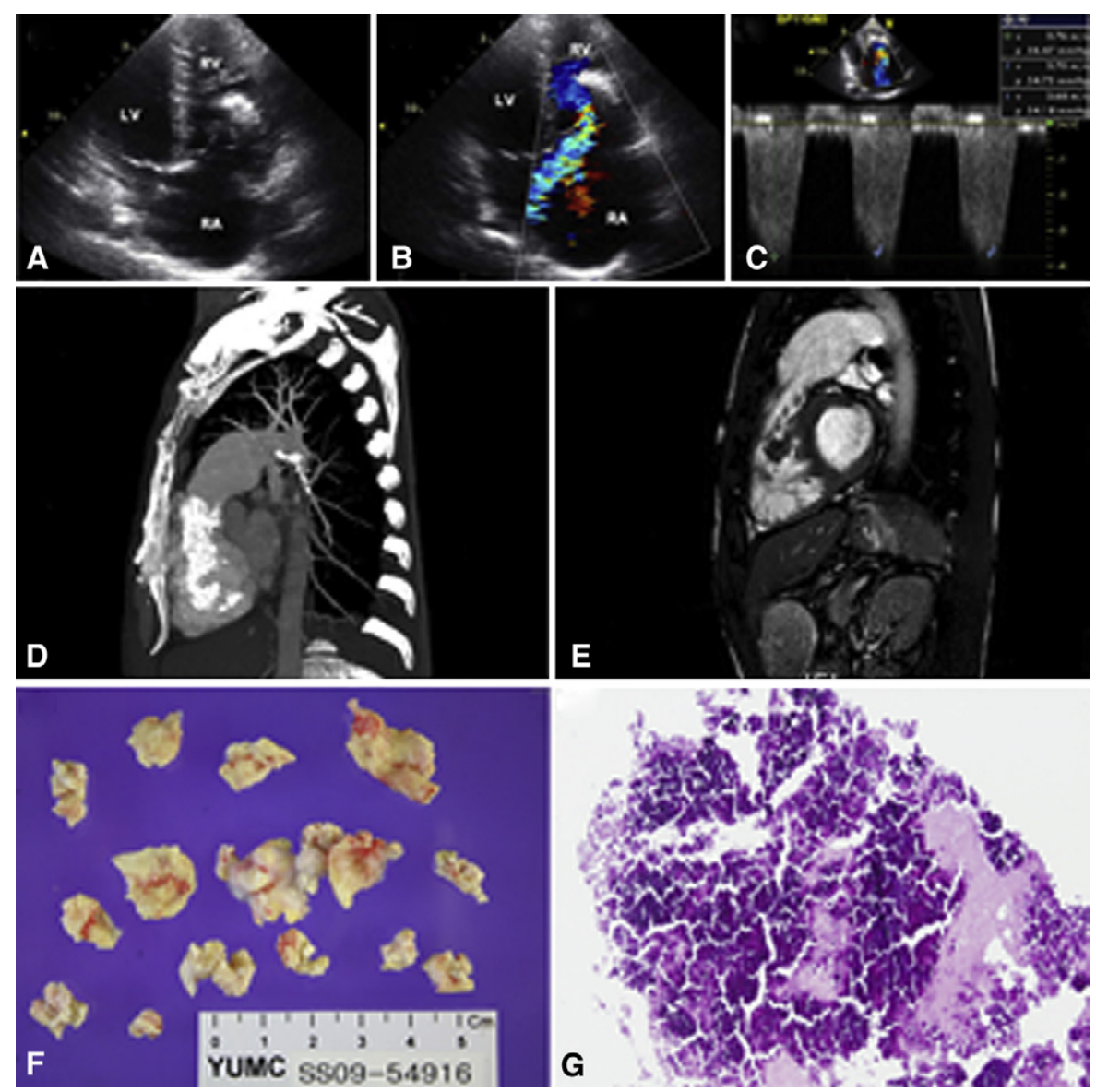

FIGURE 1. A, Two-dimensional echocardiography. B, Color Doppler echocardiography. C, Continuous-wave Doppler echocardiography. D, Chest computed tomography. E, Cardiac magnetic resonance imaging. F, Gross specimen. G, Histopathologic study. $L V$, Left ventricle; $R V$, right ventricle; $R A$, right atrium.

leads to secondary pulmonary arterial hypertension, subsequent severe tricuspid regurgitation, and congestive hepatomegaly.

This case illustrates multiple calcified right ventricular masses that probably resulted from an episode of blunt chest trauma and subsequent chronic thrombus, presenting with pulmonary embolism and severe pulmonary hypertension.

\section{References}

1. Murphy M, Sweeney M, Putnam J Jr, Walker W, Frazier O, Ott D, et al. Surgical treatment of cardiac tumors: a 25-year experience. Ann Thorac Surg. 1990;49: 612-7; discussion 617-8

2. Miller F Jr, Seward J, Gersh B, Tajik A, Mucha P Jr. Two-dimensional echocardiographic findings in cardiac trauma. Am J Cardiol. 1982;50:1022-7.

3. Reynolds C, Tazelaar H, Edwards W. Calcified amorphous tumor of the heart (cardiac CAT). Hum Pathol. 1997;28:601-6. 